\title{
Fine Mapping and Candidate Gene Analysis of Small Round Grain Mutant in Rice
}

\author{
Jeonghwan Seo $^{1 \dagger}$, Yogendra Bordiya ${ }^{1,2 \dagger}$, Chanmi Lee ${ }^{1,3}$, Hee-Jong Koh $^{1 *}$ \\ ${ }^{1}$ Department of Plant Science, Plant Genomics and Breeding Institute, Research Institute of Agriculture and Life Sciences, Seoul \\ National University, Seoul 08826, Korea \\ ${ }^{2}$ Present address: Department of Biology, Texas State University, San Marcos, TX, U.S.A \\ ${ }^{3}$ Present address: Statistics team, Data Analysis Department, Macrogen Inc., Seoul 08511, Korea
}

\begin{abstract}
Grain size and panicle architecture are important traits determining yield. Here we report a new allele of $D E P 2$ (dense and erect panicle 2) which has a pleiotropic effect on grain size/shape and panicle architecture. A small round grain and erect panicle mutant was obtained by treating japonica cultivar Hwacheong waxy with MNU (N-methyl-N-nitrosourea). Through fine mapping sequence analysis, we identified the single nucleotide substitution in the mutant, which caused splicing error of the first intron, on previously reported DEP2 locus in rice. Since the dep2-1 and dep2-2 have been reported in previous studies, we named this new allele as dep2-3. The dep2-3 mutant manifested reduced grain size and plant height, dense and erect panicle and erect plant architecture. When we crossed the mutant with wild type, panicle and grain of $F_{1}$ plant showed intermediate phenotype, indicating that dep2-3 showed incomplete dominance, which was not reported in the previous study. Scanning electron microscopy (SEM) results showed that increase in width of mutant grain was due to the increased width of glume cells. Paraffin section of peduncle revealed that outgrowth in outer layer of peduncle compared to inner layer caused the erectness of the panicle. Our results collectively indicated that the dep2-3 might play a significant role in the regulation of grain size/shape and formation of vascular bundles.
\end{abstract}

Keywords Rice, grain size, glume, erect panicle, $D E P 2$, splicing error

\section{INTRODUCTION}

Rice is the staple food for most of the human population and the second largest produced cereal crop. To increase the productivity of rice in order to meet global food requirement, we need a comprehensive understanding of the factors which control the yield and optimize them through molecular breeding and biotechnology. Amid various concerns regarding environmental protection (Byrnes 1990), the second green revolution has to be achieved using reduced chemical fertilizers. To achieve such a goal, knowledge about basic biological mechanisms has to improve further. To start with, two very important factors determining yield of rice crop are grain size and plant architecture (Sakamoto et al. 2005). Change in grain size directly affects the yield, whereas, plant architecture indirectly affects yield by affecting amount of surface area directly exposed to sun light (Kong et al. 2006).

In last decade since the advent of modern genomics technology, many QTLs regulating grain size and shape have been identified in rice (Zuo and Li 2014). GS3 is the major QTL for grain length and weight and minor QTL for grain width and thickness located on chromosome 3 (Fan et al. 2006). GS3 is supposed to be one of the most important QTLs discovered in rice so far. Function of GS3 protein could not be related to glume cell size/division yet

Received November 19, 2017; Revised November 22, 2017; Accepted November 22, 2017; Published December 1, 2017

*Corresponding author Hee-Jong Koh, heejkoh@snu.ac.kr, Tel: +82-2-880-4541, Fax: +82-2-873-2056

${ }^{\dagger}$ These authors contributed equally. 
however, study by Mao et al. (2010) linked the differential domain functions of GS3 protein to natural variation of grain size in rice. Grain size is function of glume size and grain filling, moreover, glume size is determined by the glume cell size, shape and division (Xing and Zhang 2010). Shomura et al. (2008) also reported that deletion in qSW5 (QTL for seed width on chromosome 5), increases the sink size by increasing the cell number in the outer glume surface of rice flower. The natural variation in this locus reveals that this trait was selected by humans during domestication of rice.

Dense and erect panicle is one of the desirable characteristics to breed higher yielding rice cultivars. There are several reports on genetic and molecular mechanisms controlling panicle architecture in rice such as DEP1 (dense and erect panicle 1) (Huang et al. 2009), DEP2 (dense and erect panicle 2) (Li et al. 2010), DEP3 (dense and erect panicle 3) (Qiao et al. 2011), EP (erect pose panicle) (Wang et al. 2009), EP2 (erect panicle 2) (Zhu et al. 2010) and EP3 (erect panicle 3) (Piao et al. 2009). DEPI locus is a gain-of-function mutation causing truncation of a phosphatidylethanolamine-binding protein-like domain protein. The effect of this allele is to enhance meristematic activity, resulting in a reduced length of the inflorescence internode, an increased number of grains per panicle. $E P$ is also governed by single dominant gene. Although both $E P$ and $D E P 1$ reduce the grain size but greatly enhance yield by increasing the number of grain per panicle owing to increase in number of secondary branches in case of $E P$ and number of primary and secondary branches both in case of DEP1. Zhou et al. (2009) reported qPE9-1 regulating erect panicle and plant architecture is said to be related with domestication of rice. It is proposed that the panicle erectness trait resulted from a natural random loss-offunction mutation for the qPE9-1 gene and has subsequently been the target of artificial selection during japonica rice breeding.

Here, we report the characterization and fine mapping of a small round grain mutant in rice. We identified the candidate gene of the mutant which is a new allele of previously reported $D E P 2$ locus. Phenotypic characterization and allelic relationship were also investigated.

\section{MATERIALS AND METHODS}

\section{Plant materials}

The dense and erect panicle 2-3 (dep2-3) mutant was obtained by treating Korean japonica cultivar Hwacheong waxy with MNU (N-methyl-N-nitrosourea). The mutant was selected in $\mathrm{M}_{2}$ generation and was fixed through selfing in successive generations. To genetically map the mutated gene, mapping population was developed by crossing mutant (dep2-3; japonica background) with Milyang 23 (Korean Tongil-type). $\mathrm{F}_{2}$ and $\mathrm{F}_{3}$ population were grown in green house and field respectively and these populations were used to fine map the candidate gene. Mutant parent was also crossed with its wild type parent (Hwacheong waxy) and $\mathrm{F}_{2}$ population from this cross was grown in the green house along with mapping population at experimental farm of Seoul National University, Suwon, Republic of Korea during 2010-2011.

\section{Phenotype evaluation}

To distinguish wild type and mutant phenotype, grain size characteristics like grain length, grain width and grain thickness were measured using digimatic caliper (Mitutoyo, Japan) and analyzed using Statistical Analysis System (SAS) program. Other agronomics traits like culm length, panicle length, panicle number per plant, spikelet fertility and 1000 grain weight were measured and statistically analyzed.

\section{Histological analysis}

Paraffin-embedded peduncle tissue sections were prepared following the methods described by (Piao et al. 2009) with slight modifications. Panicles were harvested at ripening stage. The peduncle was cut $1 \mathrm{~cm}$ in length from the panicle node and fixed in FAA solution ( $50 \%$ ethanol, $5 \%$ acetic acid, $3.7 \%$ formaldehyde) and stored at $4{ }^{\circ} \mathrm{C}$ for 1 day. The fixed peduncles were dehydrated with $70-100 \%$ ethanol and cleared by $25-100 \%$ xylene solution. For paraffin infiltration, the cleaned peduncle samples were soaked in the solution series of $25-100 \%$ paraffin at $55^{\circ} \mathrm{C}$. The infiltrated sample was embedded in a paraffin block and then cut into $9 \mu \mathrm{m}$ sections using a microtome (MICROM Lab, Walldorf, Germany) and mounted on 
glass slides and dried at $42^{\circ} \mathrm{C}$ for 1 day. The sections were stained in $1 \%$ safranin $/ 30 \%$ ethanol and mounted in Canada balsam. The cross sections of peduncle were observed by optical microscopy at 56X magnification.

For scanning electron microscopy (SEM), samples of rice spikelet at the mature stage were fixed overnight at $4{ }^{\circ} \mathrm{C}$ in FAA. After dehydration in a graded ethanol series and substitution with isoamyl acetate, the samples were critical-point dried, sputter coated with gold, and observed under a scanning electron microscope SUPRA 55VP (Carl Zeiss, Germany).

\section{Genetic mapping}

A total of $980 \mathrm{~F}_{2}$ and $\mathrm{F}_{3}$ plants from the cross between dep2-3 mutant and Milyang 23 were used for fine mapping. Genomic DNA were extracted from leaves using the CTAB method (Second and McCouch 1994). Eight mutants and eight wild type $\mathrm{F}_{2}$ plants were selected and an equal amount of DNA from each of the eight plants were pooled into a single sample for bulked-segregant analysis (BSA) (Michelmore et al. 1991). After BSA, fine mapping was conducted with neighboring STS markers, which were developed by based on the differences in DNA sequences between indica and japonica (Chin et al. 2007). Nine STS markers were developed additionally for fine mapping based on the available rice genome sequence data at http://www.ncbi.nlm.nih.gov/, http://www.gramene.org and http://www.rgp.dna.affrc.go.jp/. Primer sequences of 9 STS markers are listed in Table 1. PCR was performed in a reaction volume of $20 \mu \mathrm{L}$ containing $40 \mathrm{ng}$ of template DNA, $0.2 \mu \mathrm{M}$ of each primer, $200 \mu \mathrm{M}$ of each dNTP, $1 \mathrm{X}$
PCR buffer and $0.5 \mathrm{U}$ of Taq DNA polymerase. Amplifications were carried out in a PTC100 96U Thermocycler (MJ Research, Reno, NV, USA) in the following sequence: 5 minutes at $94^{\circ} \mathrm{C}$, followed by 35 cycles of 1 minute at $94^{\circ} \mathrm{C}, 1$ minute at $55^{\circ} \mathrm{C}, 1$ minute at $72^{\circ} \mathrm{C}$, and 5 minutes at $72^{\circ} \mathrm{C}$ for a final extension. $\mathrm{PCR}$ products were separated in $3 \%$ agarose gel and visualized by ethidium bromide staining.

\section{Sequence analysis of the candidate gene}

The full-length genomic DNA sequences of candidate gene were determined by dividing the genes into several overlapping segments. Specific PCR primers for each segment were used to amplify genomic DNA from wild type and mutant. PCR products were purified using a PCR purification kit (Bioneer, Deajeon, Korea) for TA cloning. Purified PCR product was introduced into the pGEM-T Easy Vector (Promega, Madison, WI, USA) and transformed into the E. coli strain DH5 $\alpha$. Sequencing of genomic inserts was performed using an ABI Prism 3730 XL DNA Analyzer (Applied Biosystems, Foster City, CA, USA). Sequence alignment was performed with the BLAST network services in National Center for Biotechnology Information (NCBI) and the European Bioinformatics Institute. The results of sequencing were aligned with wild type parent using CodonCode Aligner software (CodonCode Corporation, USA).

\section{CAPS maker analysis and RT-PCR}

For cleaved amplified polymorphic sequence (CAPS) analysis, PCR amplification with the primer set

Table 1. STS markers used in fine mapping of the dep2-3.

\begin{tabular}{lllc}
\hline \hline Marker & \multicolumn{1}{c}{ Forward primer $\left(5^{\prime}-3^{\prime}\right)$} & \multicolumn{1}{c}{ Reverse primer (5'-3') } & Product size (bp) \\
\hline S02458 & CAAGCTCTCTATGCATGGCTAA & GCTGTTCCATGAGATACACTACAAA & 140 \\
S02468 & ACGGCGAGAAGGATAAGGAT & GCTAGCCTACACTCCGTTGG & 194 \\
S02471 & CGGGTCGTTGTCTTCTCAT & TGTGGCGGCTAATCTGATCT & 179 \\
S02497 & CCGCAAGATGATGGTAACCT & TCGGATCCTCGGATGTAAAG & 201 \\
S02519 & TTCCTGTCAATATGCGGTATCA & AATATCGAACGAAGCACATCC & 138 \\
S02535 & GCGTTGTCAGCTGTCCTCTT & TCGGGCATCACTAGATACCA & 195 \\
S02541 & CTTTGCACTCTCAGCCCATT & CAAGTTTGGGGTCATTTG & 159 \\
S02548 & CCCTACGAGGAGGAGATGAG & AGGTAACCGAGCAGCAACAT & 213 \\
S02552 & CCCAGAACTGGATGATGGTT & TGCCCATACGGAGAGAAAAG & 186 \\
\hline
\end{tabular}


Nru1CAPS-F (5'-GAGGAGAGCTTGGGGTTCTT-3') and Nru1CAPS-R (5'-GTGCCCTTGCTGAACCAC-3') were used. After PCR, each product $(5 \mu \mathrm{L})$ was digested with $N r u$ I in a total volume of $15 \mu \mathrm{L}$ at $37^{\circ} \mathrm{C}$ for 2 hours. After digestion, $5 \mu \mathrm{L}$ of each digest was visualized on a $2.5 \%$ agarose gel.

Total RNA was extracted from spikelet of wild type and mutant at the heading stage, using TRIzol isolation reagent (Invitrogen, USA), according to the manufacturer's protocol. The RNA was pre-treated with DNase I (Takara, Japan), following the manufacturer's instruction. Total RNA was reverse-transcribed using an M-MLV reverse transcriptase kit (Promega, USA). The synthesized fi rst-strand cDNAs was used for PCR analysis with dep2-specific primers for target region amplification, dep2-RT-F (5'-ACTTCGCGCTCTTCCAGCTCT-3') and dep2-PR-R (5'-AGGTGCGCCACGAACGGCTT-3').

\section{RESULTS}

\section{Characterization of the dep2-3 mutant}

The dep 2-3 mutant showed small round grain phenotype compared to wild type (Fig. 1A). The average grain length of wild type was $5.73 \mathrm{~mm}$ whereas that of mutant type was $4.83 \mathrm{~mm}$. Unlike grain length, grain width and grain thickness were higher in case of mutant type compared to wild type. The ratio of grain length to width was 1.74 in wild type whereas that of mutant was 1.39 (Table 2). This categorizes wild type grain into semi round and mutant grain into round shape category. The plant height of mutant was observed to be shorter than that of wild type and the overall architecture of the plant was erect compared to wild type (Fig. 1C). Due to reduction in grain length, the 1000 grain weight was significantly reduced in mutant $(21 \mathrm{~g})$ compared to wild type (23.3 g). Reduction in panicle length was also observed with the average length of wild type panicle being $19 \mathrm{~cm}$ compared to $14.10 \mathrm{~cm}$ that of mutant type. Also, decrease in the spikelet fertility was observed in mutant. However, there was no significant difference found in number of panicles per plant (Table 2).

\section{Histological analysis}

The change in glume cell size directly affects the grain size. Therefore, to observe change in glume cell size we did scanning electron microscopic observation of outer glume
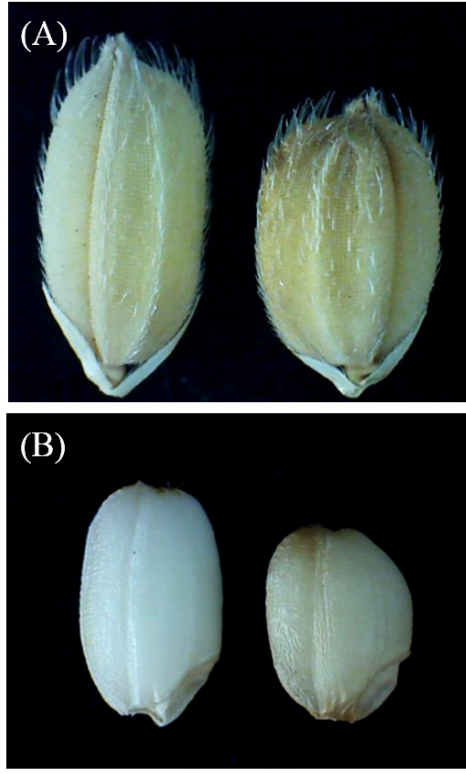

WT

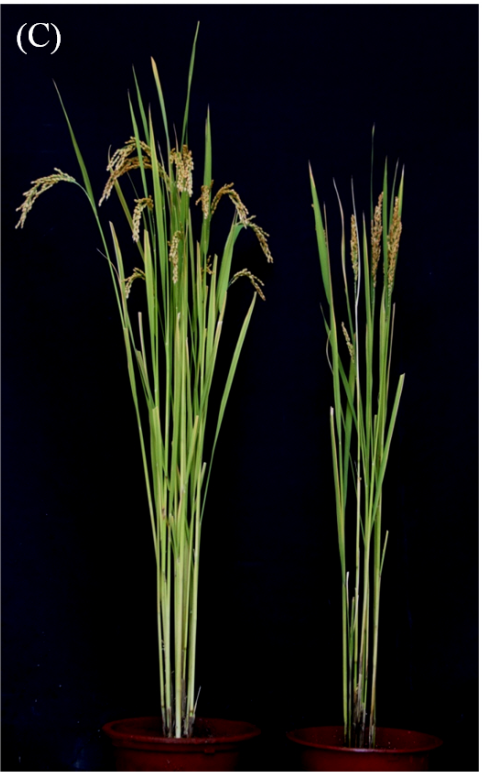

WT
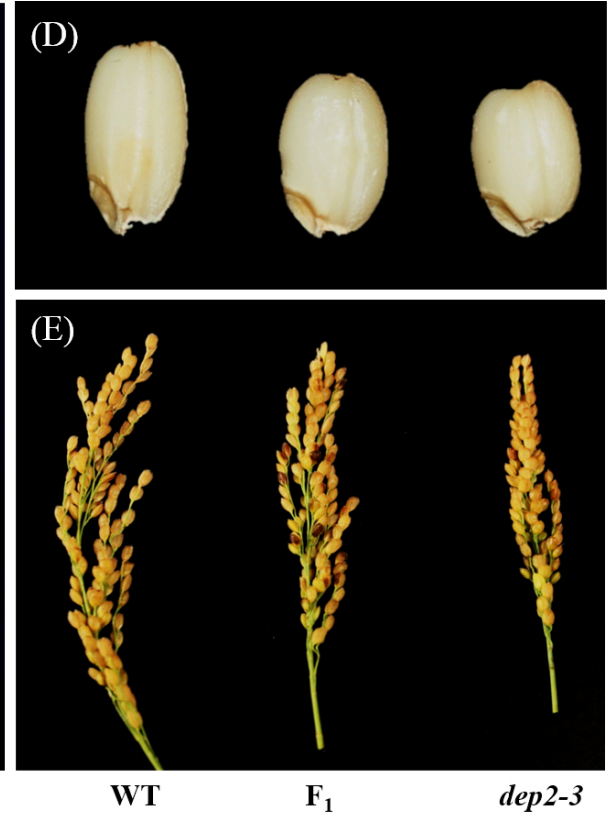

Fig. 1. Phenotype of wild type and dep2-3 mutant. Phenotype of grain (A), brown rice (B, D), plant (C) and panicle (E) at the mature grain stage. 
Table 2. Statistical analysis of agronomic traits and grain characteristics of wild type, mutant and $F_{1}$.

\begin{tabular}{|c|c|c|c|c|c|c|c|c|c|}
\hline Traits & $\mathrm{CL}^{\mathrm{z})}(\mathrm{cm})$ & PL (cm) & $\mathrm{PN}$ & SF (\%) & TGW (g) & GW (mm) & GL (mm) & GT (mm) & LWR \\
\hline Wild-type & $87.00 \pm 4.92$ & $19.00 \pm 0.94$ & $9.50 \pm 2.12$ & $96.61 \pm 0.95$ & $23.30 \pm 0.02$ & $3.29 \pm 0.09$ & $5.73 \pm 0.22$ & $2.08 \pm 0.06$ & $1.74 \pm 0.09$ \\
\hline Mutant & $72.50 \pm 3.85$ & $14.10 \pm 1.45$ & $8.50 \pm 1.5$ & $90.45 \pm 2.45$ & $21.00 \pm 0.03$ & $3.47 \pm 0.13$ & $4.83 \pm 0.14$ & $2.20 \pm 0.1$ & $1.39 \pm 0.06$ \\
\hline $\mathrm{F}_{1}$ & $86.00 \pm 2.2$ & $16.62 \pm 0.98$ & $9.30 \pm 1.5$ & $96.00 \pm 1.0$ & $21.93 \pm 0.02$ & $3.39 \pm 0.12$ & $5.47 \pm 0.23$ & $2.15 \pm 0.06$ & $1.61 \pm 0.08$ \\
\hline \multicolumn{10}{|l|}{ Difference } \\
\hline WT vs Mutant & $* *$ & $* *$ & NS & $* *$ & $* *$ & $* *$ & $* *$ & $* *$ & $* *$ \\
\hline WT vs $F_{1}$ & NS & $* *$ & NS & NS & $* *$ & $* *$ & $* *$ & $* *$ & $* *$ \\
\hline Mutant vs $F_{1}$ & $* *$ & $* *$ & NS & $* *$ & $*$ & $*$ & $* *$ & $*$ & $* *$ \\
\hline
\end{tabular}

z) Abbreviations indicate CL: Culm Length, PL: Panicle Length, PN: Panicle Number per plant, SF: Spikelet Fertility, TGW: 1000 Grain Weight, GW: Grain Width, GL: Grain Length, GT: Grain Thickness, LWR: Length Width Ratio of grain. $*$ and $* *$ indicate significant at $5 \%$ and $1 \%$ probability level, respectively.

(B)
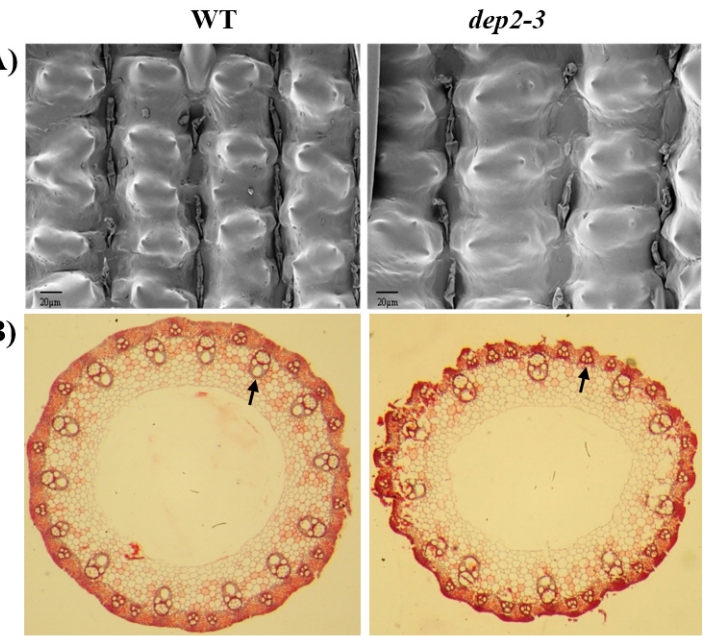

(C)

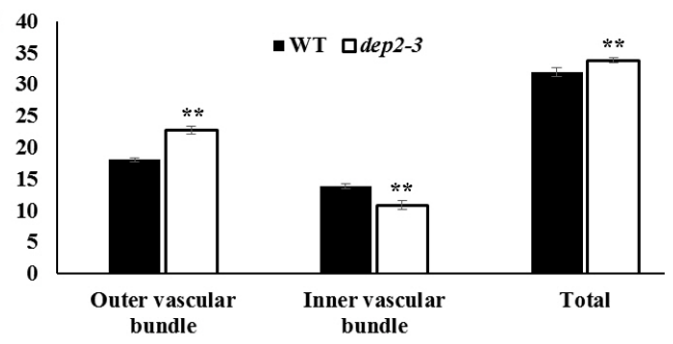

Fig. 2. Histological analysis of wild type and dep2-3 mutant. (A) Scanning electron microscopic view of the surface of glumes. (B) Cross section of the peduncle. Arrows showing inner large vascular bundle and outer small vascular bundle in wild type and dep2-3, respectively. (C) Comparison of the number outer, inner and total vascular bundles. $* *$ indicates significant at $1 \%$ probability level.

surface with $1000 \mathrm{X}$ magnification. A clear increase in the cell width was observed in case of mutant compared to wild type (Fig. 2A). This increase in cell width explains increase in the grain width in mutant.

To observe the changes at histological level in panicle, we performed paraffin sectioning of the peduncle (Fig. 2B). Distinct difference could be observed in the number of vascular bundles. The number of inner large vascular bundle was significantly higher in wild type whereas, the number of outer small vascular bundles was significantly higher in mutant. The total number of vascular bundle was found to be significantly higher in mutant (Fig. 2C). This result shows that in the mutant, there is more growth in outer layer of peduncle compared to inner layer which indicates that the erectness of the panicle.

\section{Genetic analysis of dep2-3}

To know whether dep2-3 behaves as recessive or dominant gene, we crossed mutant with its original parent. Interestingly, $F_{1}$ plant showed semi erect panicle and intermediate grain characteristics between wild type and mutant with statistically significant difference (Fig. 1D, 1E, Table 2). Through this data we concluded that dep2-3 shows incomplete dominance. We also measured grain length and width of all $F_{3}$ plants derived from cross between dep2-3 and Milyang 23, and evaluated the frequency distribution pattern. Both grain length and length width ratio showed normal distribution (Fig. 3).

\section{Fine mapping of $\operatorname{dep} 2-3$}

To genetically map the dep2-3 we performed bulked segregant analysis (BSA) using STS markers. Among total 80 markers which covered all chromosomes, two markers, 

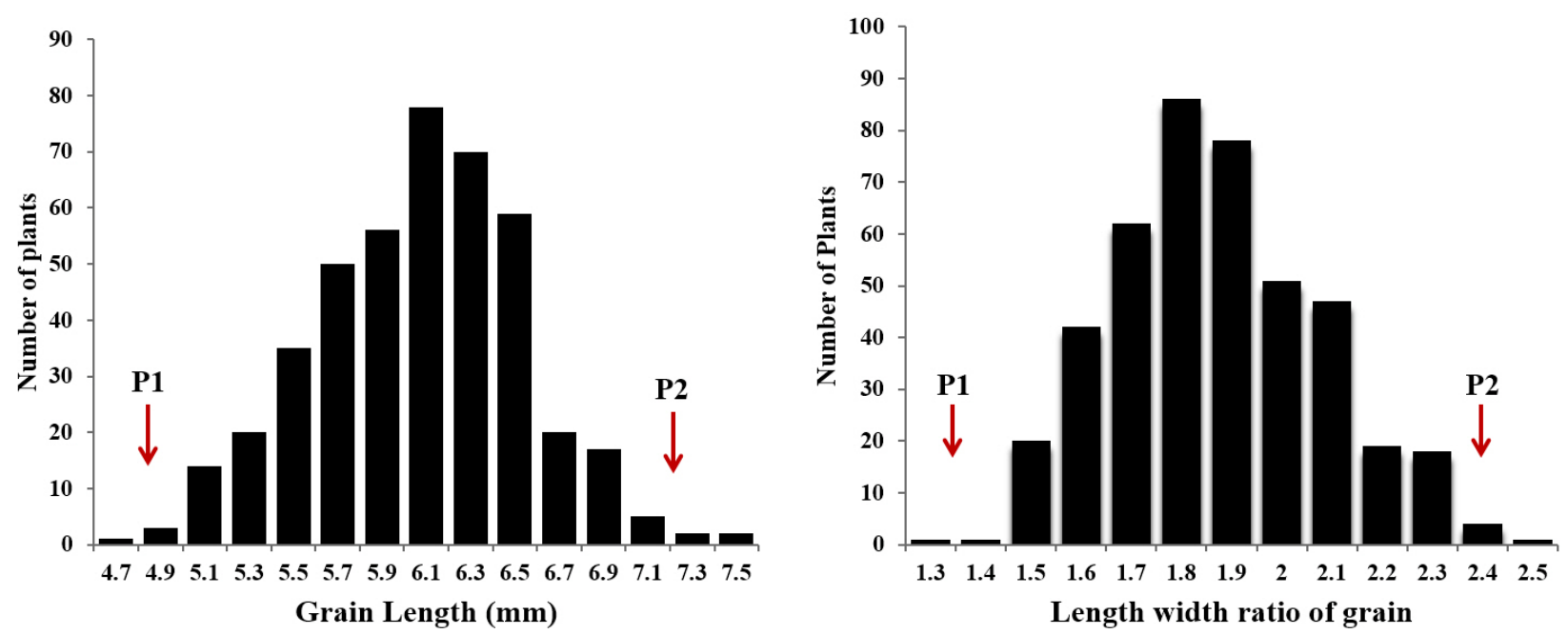

Fig. 3. Frequency distribution of grain length and length width ratio in $\mathrm{F}_{2}$ population derived from cross between dep2-3 mutant and Milyang 23. P1 and P2 indicate the value of parents; dep2-3 (P1) and Milyang 23 (P2), respectively.

BSA

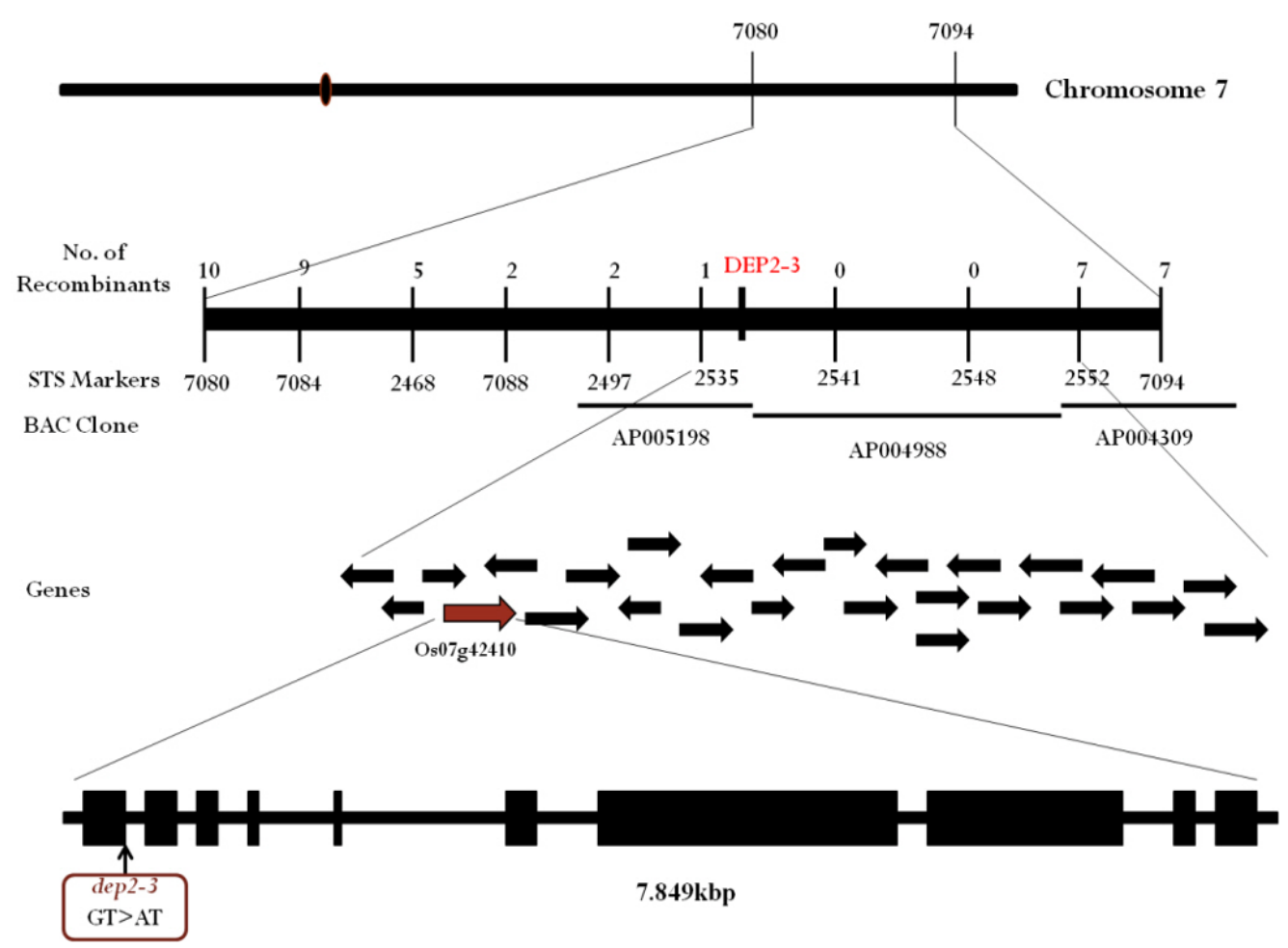

Fig. 4. Genetic mapping of dep2-3 mutant. Location of dep2-3 was found on BAC clone AP005198. Sequencing result of LOC_Os07g42410 revealed point mutation ( $\mathrm{G}$ to A) at the 5' end splicing site of first intron.

S07080 and S07094 on long arm of chromosome 7 demonstrated significant polymorphism between bulked DNA samples of wild and mutant type plants. To further narrow down the candidate region additional 9 STS markers were designed (Table 1). Through additional markers, we narrowed down the candidate region to 
$168.75 \mathrm{kbp}$ between marker S02535 and S02552 (Fig. 4). Through rice genome annotation database (http://rice. plantbiology.msu.edu/cgi-bin/gbrowse/rice/) we found that this candidate region of $168.75 \mathrm{kbp}$ had a total of 26 genes. Among 26 genes the LOC_Os07g42410 was reported to be related with erect panicle phenotype in maize and rice (DEP2). After sequencing this gene, we found a point mutation ( $\mathrm{G}$ to $\mathrm{A})$ at the 5 ' splicing site of first intron (Fig. 4).

\section{Candidate gene analysis}

To confirm the point mutation, CAPS marker with $\mathrm{Nru} 1$ restriction enzyme site (TCGCGA) was designed. Seven mutant type and 7 wild type plants were selected from $\mathrm{F}_{3}$ population and PCR was performed using CAPS marker which, after digestion with $N r u \mathrm{I}$, will cut the mutant sequence. A clear co-segregation was observed between wild type and mutant type plants from $\mathrm{F}_{3}$ population (Fig. 5A).

In case of eukaryotes, mRNA has introns and exons. Introns are spliced off before translation of mRNA. Intron has a specific sequence GU at its 5' end and AG at its 3' end. In dep2-3 mutant this $\mathrm{G}$ has been mutated to $\mathrm{A}$. Spliceosome which binds at this site and helps in splicing off intron will not be able to do so after mutation. To identify the splicing form of mutation site, we conducted RT-PCR analysis of wild type and mutant using primers designed on first exon and second exon. In wild type first intron ( $82 \mathrm{bp}$ ) was successfully spliced off, but in mutant first intron was not spliced off (Fig. 5B). This result suggests that point mutation on 5 ' splicing site of first intron in dep2-3 cause the splicing error and might also lead to frameshift mutation.

\section{DISCUSSION}

One common phenomenon which could be distinctly observed in almost previous and also in current study in erect panicle mutants is that, almost erect panicle mutants manifest small grain phenotype and slight reduction in plant height. This observation clearly shows that almost these genes $E P 2, E P 3, D E P 1, q P E 9-1$ and $D E P 2$ directly or indirectly affects cell size/shape and/or cell division which changes plant and panicle architecture. Previous study on dep2 (Li et al. 2010) reported that the expression level of cell cycle related genes like $C y c B 1 ; 1, C y c B 2 ; 1$, $C y c B 2 ; 2, C y c D 3 ; 1$, and $C D K B 2 ; 1$ was reduced in mutant compared to wild type while cell size remained unchanged. However, in present study we observed the change in cell shape in mutant glume (Fig. 2A), this indicates that DEP2 has pleiotropic effect on the cell division and cell shape. Genes affecting plant architecture also tend to affect the grain yield by affecting glume size. Since the overall architecture of plant is shaped by cell division, cell size and its shape, the genes which affect plant height and panicle characteristics also have a substantial effect on the grain size. Therefore, it is important to understand the mechanism involved in plant architecture at molecular level.

Erect panicle phenotype is the result of decrease in
(A)

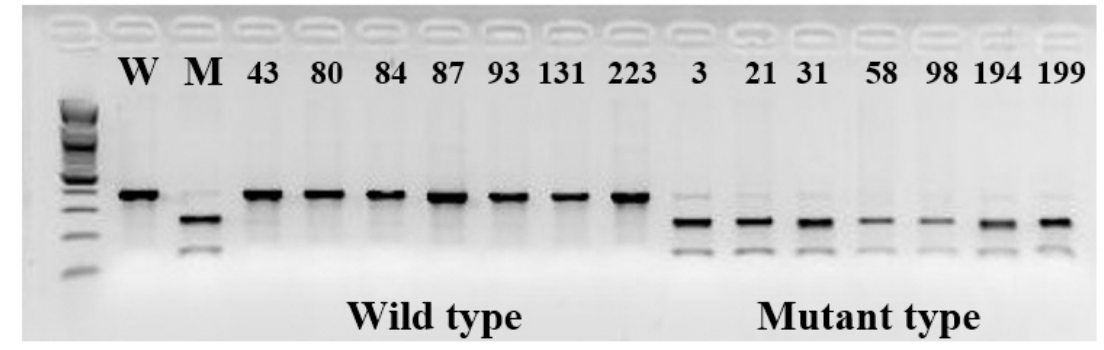

(B)

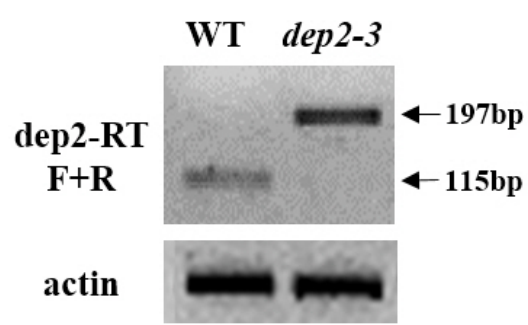

Fig. 5. Candidate gene analysis of dep2-3. (A) Co-segregation test wild and mutant type plants from $\mathrm{F}_{3}$ population using CAPS marker with $N r u$ I restriction enzyme. (B) RT-PCR analysis of wild type and mutant using primers designed on first exon and second exon. Size of first intron is $82 \mathrm{bp}$ and expected PCR product size using wild type cDNA is $115 \mathrm{bp}$. 
length of rachis, primary and secondary branch, this might be due to decrease in cell size or due decreased cell division, moreover, as mentioned earlier, this also affects grain size negatively. However, decrease in grain size does not always lead to reduction in grain yield, according to the report by Huang et al. (2009), depl enhances the grain yield by increasing number of grains per panicle. Therefore, to achieve maximum yield there is need to optimize the plant architecture, grain size, shape and number.

In the histological analysis of peduncle, higher number of vascular bundle in mutant, (specifically small, outer vascular bundle) indicates more growth in outer cell layers. This makes the stem and panicle stronger and is responsible for the erectness. Further proteomic study has to be done to know if there is any relation of dep 2 protein with plant hormones like auxin, cytokinin or brassinosteroids. Since plant hormones affect the cell division and shape and growth, there might be some common link between dep2 protein and hormone pathway.

First erect panicle variety named Guihuahuang was released in China in 1960's, since then breeders have developed many erect panicle varieties which have contributed to increase in rice production in China. Identification of genes related with erect panicle and their alleles in various japonica and indica cultivars will further strengthen the rice breeding programs.

\section{ACKNOWLEDGEMENTS}

This work was supported by a grant from the Next-Generation BioGreen 21 Program (Plant Molecular Breeding Center No. PJ01102401), Rural development Administration, Republic of Korea.

\section{REFERENCES}

Byrnes B. 1990. Environmental effects of N fertilizer useAn overview. Nutrient Cycling in Agroecosystems 26: 209-215.

Chin JH, Kim JH, Jiang W, Chu SH, Woo MO, Han L, et al.
2007. Identification of subspecies-specific STS markers and their association with segregation distortion in rice (Oryza sativa L.). J. Crop Sci. Biotech. 10: 175-184.

Fan C, Xing Y, Mao H, Lu T, Han B, Xu C, et al. 2006. GS3, a major QTL for grain length and weight and minor QTL for grain width and thickness in rice, encodes a putative transmembrane protein. Theor. Appl. Genet. 112: 11641171.

Huang X, Qian Q, Liu Z, Sun H, He S, Luo D, et al. 2009. Natural variation at the $D E P 1$ locus enhances grain yield in rice. Nat. Genet. 41: 494-497.

Kong FN, Wang JY, Zou JC, Shi LX, Jin M, Xu ZJ, et al. 2006. Molecular tagging and mapping of the erect panicle gene in rice. Mol. Breed. 19: 297-304.

Li F, Liu W, Tang J, Chen J, Tong H, Hu B, et al. 2010. Rice DENSE AND ERECT PANICLE 2 is essential for determining panicle outgrowth and elongation. Cell Res. 20: 838-849.

Mao H, Sun S, Yao J, Wang C, Yu S, Xu C, et al. 2010. Linking differential domain functions of the GS3 protein to natural variation of grain size in rice. Proc. Natl. Acad. Sci. U.S.A. 107: 19579-19584.

Michelmore RW, Paran I, Kesseli R. 1991. Identification of markers linked to disease-resistance genes by bulked segregant analysis: a rapid method to detect markers in specific genomic regions by using segregating populations. Proc. Natl. Acad. Sci. U.S.A. 88: 9828-9932.

Piao R, Jiang W, Ham TH, Choi MS, Qiao Y, Chu SH, et al. 2009. Map-based cloning of the ERECT PANICLE 3 gene in rice. Theor. Appl. Genet. 119: 1497-1506.

Qiao Y, Piao R, Shi J, Lee SI, Jiang W, Kim BK, et al. 2011. Fine mapping and candidate gene analysis of dense and erect panicle 3, DEP3, which confers high grain yield in rice (Oryza sativa L.). Theor. Appl. Genet. 122: 1439-1449.

Sakamoto T, Morinaka Y, Ohnishi T, Sunohara H, Fujioka S, Ueguchi-Tanaka M, et al. 2005. Erect leaves caused by brassinosteroid deficiency increase biomass production and grain yield in rice. Nat. Biotechnol. 24: 105-109.

Second G, McCouch R. 1994. Saturated molecular map of the rice genome based on an interspecific backcross population. Genetics 138: 1251-1274.

Shomura A, Izawa T, Ebana K, Ebitani T, Kanegae H, Konishi S, et al. 2008. Deletion in a gene associated with grain size increased yields during rice domestication. Nat. 
Genet. 40: 1023-1028.

Wang J, Nakazaki T, Chen S, Chen W, Saito H, Tsukiyama T, et al. 2009. Identification and characterization of the erect-pose panicle gene EP conferring high grain yield in rice (Oryza sativa L.). Theor. Appl. Genet. 119: 85-91.

Xing Y, Zhang Q. 2010. Genetic and molecular bases of rice yield. Annu. Rev. Plant Biol. 61: 421-442.

Zhou Y, Zhu J, Li Z, Yi C, Liu J, Zhang H, et al. 2009.

Deletion in a quantitative trait gene $q P E 9-1$ associated with panicle erectness improves plant architecture during rice domestication. Genetics 183: 315-324.

Zhu K, Tang D, Yan C, Chi Z, Yu H, Chen J, et al. 2010. Erect panicle 2 encodes a novel protein that regulates panicle erectness in indica rice. Genetics 184: 343-350.

Zuo J, Li J. 2014. Molecular genetic dissection of quantitative trait loci regulating rice grain size. Annu Rev Genet. 48: 99-118. 